Louisiana State University

LSU Digital Commons

Faculty Publications

Department of Biological Sciences

6-1-2009

\title{
High-throughput single cell arrays as a novel tool in biopreservation
}

\author{
Kenneth L. Roach \\ Massachusetts General Hospital \\ Kevin R. King \\ Massachusetts General Hospital \\ Korkut Uygun \\ Massachusetts General Hospital \\ Steven C. Hand \\ Louisiana State University \\ Isaac S. Kohane \\ Harvard-MIT Health Sciences and Technology
}

See next page for additional authors

Follow this and additional works at: https://digitalcommons.Isu.edu/biosci_pubs

\section{Recommended Citation}

Roach, K., King, K., Uygun, K., Hand, S., Kohane, I., Yarmush, M., \& Toner, M. (2009). High-throughput single cell arrays as a novel tool in biopreservation. Cryobiology, 58 (3), 315-321. https://doi.org/10.1016/ j.cryobiol.2009.03.001

This Article is brought to you for free and open access by the Department of Biological Sciences at LSU Digital Commons. It has been accepted for inclusion in Faculty Publications by an authorized administrator of LSU Digital Commons. For more information, please contact ir@lsu.edu. 
Authors

Kenneth L. Roach, Kevin R. King, Korkut Uygun, Steven C. Hand, Isaac S. Kohane, Martin L. Yarmush, and Mehmet Toner 


\title{
High Throughput Single Cell Arrays as a Novel Tool in Biopreservation
}

\author{
Kenneth L. Roach ${ }^{1,2}$, Kevin R. King ${ }^{1,2}$, Korkut Uygun ${ }^{1}$, Steven C. Hand ${ }^{3}$, Isaac S. \\ Kohane $^{2,4}$, Martin L. Yarmush ${ }^{1,2}$, and Mehmet Toner ${ }^{1,2}$ \\ ${ }^{1}$ Center for Engineering in Medicine, BioMEMS Resource Center, Massachusetts General Hospital, \\ Harvard Medical School, and Shriners Hospital for Children, Boston, Massachusetts, USA \\ ${ }^{2}$ Harvard-MIT Division of Health Sciences and Technology, Cambridge, Massachusetts, USA \\ ${ }^{3}$ Division of Cellular, Developmental and Integrative Biology, Department of Biological Sciences, \\ Louisiana State University, Baton Rouge, Louisiana, USA \\ ${ }^{4}$ Informatics Program, Children's Hospital Boston and Harvard Medical School, Boston, \\ Massachusetts, USA
}

\begin{abstract}
Microwell array cytometry is a novel high-throughput experimental technique that makes it possible to correlate pre-stress cell phenotypes and post-stress outcomes with single cell resolution. Because the cells are seeded in a high density grid of cell-sized microwells, thousands of individual cells can be tracked and imaged through manipulations as extreme as freezing or drying. Unlike flow cytometry, measurements can be made at multiple time points for the same set of cells. Unlike conventional image cytometry, image analysis is greatly simplified by arranging the cells in a spatially defined pattern and physically separating them from one another. To demonstrate the utility of microwell array cytometry in the field of biopreservation, we have used it to investigate the role of mitochondrial membrane potential in the cryopreservation of primary hepatocytes.
\end{abstract}

Even with optimized cryopreservation protocols, the stress of freezing almost always leads to dysfunction or death in part of the cell population. To a large extent, cell fate is dominated by the stochastic nature of ice crystal nucleation, membrane rupture, and other biophysical processes, but natural variation in the initial cell population almost certainly plays an important and under-studied role. Understanding why some cells in a population are more likely to survive preservation will be invaluable for the development of new approaches to improve preservation yields.

For this paper, primary hepatocytes were seeded in microwell array devices, imaged using the mitochondrial dyes Rh123 or JC-1, cryopreserved for up to a week, rapidly thawed, and checked for viability after a short recovery period. Cells with a high mitochondrial membrane potential before freezing were significantly less likely to survive the freezing process, though the difference in short term viability was fairly small. The results demonstrate that intrinsic cell factors do play an important role in cryopreservation survival, even in the short term where extrinsic biophysical factors would

(C) 2009 Elsevier Inc. All rights reserved.

Correspondence to: Mehmet Toner, Ph.D., Center for Engineering in Medicine, Massachusetts General Hospital, Bldg 114, 16th Street, Charlestown, MA 02129, Phone: 617-724-5336, Email: E-mail: mtoner@hms.harvard.edu.

Publisher's Disclaimer: This is a PDF file of an unedited manuscript that has been accepted for publication. As a service to our customers we are providing this early version of the manuscript. The manuscript will undergo copyediting, typesetting, and review of the resulting proof before it is published in its final citable form. Please note that during the production process errorsmaybe discovered which could affect the content, and all legal disclaimers that apply to the journal pertain. 
be expected to dominate. We believe that microwell array cytometry will be an important tool for a wide range of studies in biopreservation and stress biology.

\section{Keywords}

array cytometry; microfabrication; biopreservation; cryopreservation; hepatocytes; mitochondria; membrane potential

\section{Introduction}

There is a significant need for high-throughput and high-content tools in biopreservation and stress biology, particularly for the investigation and discovery of new ways to improve preservation outcomes [17]. In biopreservation, even with highly optimized protocols, the extreme stress of freezing desiccation almost always leads to dysfunction or death in a significant fraction of the cell population. It is well known that cell fates are determined in part by the stochastic nature of ice crystal nucleation and other biophysical processes [7]. Less well understood is the extent to which metabolic, bioenergetic, transcriptional, and genetic variation in the initial cell population plays a significant role. By understanding how the initial state of each cell affects its response to preservation, it will be possible to develop new targets for intervention.

Cell-to-cell variation can be quite significant, particularly with primary cells that have been isolated from their native tissue environment. Primary hepatocytes, for instance, vary widely in their metabolic behavior, with either enhanced detoxification or ammonia removal functions depending on their locations in the sinusoids [14]. Mitochondrial activity, metabolic state, energy reserves, position the cell cycle, and membrane composition could all potentially affect preservation outcomes, but few good tools exist to examine these effects at the single cell level.

Microwell array cytometry is a powerful new experimental technique that can be used to correlate pre-stress cell phenotypes and post-stress outcomes with single cell resolution. The system discussed in this paper was designed in our research group as part of a general effort to develop high-throughput and high-content tools for single cell biology [21]. Because the cells are seeded in rugged, high density grids of cell-sized microwells, thousands of individual cells can be tracked and imaged through manipulations as extreme as freezing or drying. Automated image analysis software makes working with the data as straightforward as in flow cytometry.

Microwell array cytometry also has a number of significant advantages over conventional approaches. Unlike flow cytometry, measurements can be made at multiple time points for the same set of cells. Unlike image cytometry with random cell seeding, image analysis is greatly simplified by arranging the cells in a spatially defined pattern and physically separating them from one another. To demonstrate the utility of microwell array cytometry in the field of biopreservation, we have employed it to investigate the role of mitochondrial membrane potential $(\Delta \Psi)$ in the cryopreservation of primary hepatocytes.

\section{Importance of Mitochondria in Cryopreservation Outcomes}

Resistance to one or more extreme stresses can be found in many organisms, though such resistance is by no means common or universal. Even closely related organisms can have widely different tolerance levels. The adaptations allowing for tolerance are subtle and often the result of many small changes to metabolic and stress response systems rather than the production of a single protective element [26]. Even in cases where such a protective element obviously exists, such as trehalose in rotifers and brine shrimp or circulating glucose in wood 
frogs, the use of these compounds to protect otherwise intolerant cells has been only modestly successful [3]. In other cases, the presence of a protective compound has been shown to be neither necessary nor sufficient for tolerance in the original organism [29]. It has become clear that further progress in biopreservation will require a better understanding of metabolic, bioenergetic, and transcriptional systems, both the protective ones employed by the cells of tolerant organisms and the detrimental ones activated in other cell types. Given the complexity of these systems, bioinformatics and systems biology approaches will be invaluable in this pursuit.

Mitochondria are known to play a key role in metabolism, bioenergetics, calcium homeostasis, and cell death pathways [15]. Naturally tolerant organisms are also known to possess a number of mitochondrial adaptations that are critical for survival in the stressed state [9-11]. Many such organisms enter a state of metabolic stasis and reduced mitochondrial activity in anticipation of environmental stress $[8,25]$. Brine shrimp embryos, for instance, reduce their oxygen consumption by up to $92 \%$ upon entering diapause [20]. These observations suggest that having relatively quiescent mitochondria and reduced mitochondrial activity might also be protective for mammalian cells during biopreservation. If significant correlations exist between mitochondrial states and cryopreservation outcomes, novel preservation methods and treatments can be developed to adjust these states prior to cryopreservation.

The experiments of this paper are intended to investigate this idea in a powerful new way. The goal is to use microwell array cytometry system to precisely determine the extent to which the natural variation in mitochondrial membrane potential across a cell population helps determine preservation outcomes. To date, most biopreservation studies have focused on either bulk assays or small numbers of cells. The experiments described here are some of the first to use high throughput, single cell technologies in biopreservation research. By identifying the most favorable mitochondrial states before and after preservation, it may be possible to improve overall outcomes by pushing cells towards these states with various modulators of mitochondrial activity.

\section{Methods and Materials}

\section{Assembly and Coating}

Standard glass slides were cut into $2.5 \times 1.0 \mathrm{~cm}$ miniature slides intended to fit in $1.5 \mathrm{ml}$ Nalgene cryovials. Individual devices containing 16 arrays each were cut from replica molded polydimethylsiloxane (PDMS) sheets and reversibly mounted well-side-up on a miniature slide [21]. The devices were sterilized by either autoclaving after assembly or rinsing in $100 \%$ ethanol before assembly. A $1.25 \mathrm{mg} / \mathrm{ml}$ stock solution of Type I collagen was prepared as described elsewhere and diluted 1:50 in distilled water before use [5]. Each device was covered with a droplet of collagen solution and placed under vacuum for $15 \mathrm{~min}$ to improve microwell filling. The devices were then incubated for another $30 \mathrm{~min}$ at $25^{\circ} \mathrm{C}$, rinsed with media, and kept wet until seeding.

\section{Hepatocyte Seeding}

Primary rat hepatocytes were freshly isolated from female Lewis rats and resuspended at $1 \times 10^{6} \mathrm{cells} / \mathrm{ml}$ in $\mathrm{C}+\mathrm{H}$ hepatocyte culture medium [5]. Particular care was taken with the pipetting to gently break up clumps of cells and achieve a single cell suspension. Droplets of the suspension were then placed on each device and periodically recirculated by gentle pipetting to improve filling efficiency and reduce cell attachment outside of the microwells. After 5-10 minutes, excess cells were removed by rinsing with fresh media, and the devices were briefly examined under phase contrast microscopy. If loading was poor after the first round of seeding, it was repeated a second time. Once fully loaded, each device was transferred 
to a dish of fresh medium and gently agitated to remove any cells remaining on the surface of the device.

\section{Mitochondrial Staining}

The fluorescent dyes JC-1 and rhodamine 123 (Sigma Aldrich) were used to assess mitochondrial membrane potential $(\Delta \Psi)$ in the cells [4,22]. Both are lipophilic, cationic molecules that cross the mitochondrial membrane and accumulate in the negatively charged mitochondrial matrix in a potential dependent manner, with fluorescence detectable by a GFP (green) filter set. At the high concentrations achieved in polarized mitochondria, JC-1 molecules form aggregates with a different fluorescence peak than the dilute dye, detectable with a Cy3.5 (red) filter set. Rhodamine 123 does not have this property and instead tends to self-quench at very high concentrations. Both dyes were used, but in separate experiments. JC-1 provides better information about mitochondrial polarization, but covers two fluorescence channels. Rh123 is more difficult to interpret, but in future studies could be used in combination with a red fluorescent dye for multifactorial analysis.

Stock JC-1 solution was prepared by dissolving $1 \mathrm{mg}$ lyophilized JC-1 in $1 \mathrm{ml}$ dimethylsulphoxide $\left(\mathrm{Me}_{2} \mathrm{SO}\right)$. The stock solution was diluted 1:200 in $\mathrm{C}+\mathrm{H}$ culture medium before use for a final concentration of $5 \mu \mathrm{g} / \mathrm{ml}$. Stock rhodamine 123 (Rh123) solution was prepared by dissolving $10 \mathrm{mg}$ lyophilized $\mathrm{Rh} 123$ in $1 \mathrm{ml} \mathrm{Me} 2 \mathrm{SO}$. In this case, the stock was diluted 1:1000 in $\mathrm{C}+\mathrm{H}$ before use for a final concentration of $10 \mu \mathrm{g} / \mathrm{ml}$. Both stock solutions were stored at $-20^{\circ} \mathrm{C}$. Seeded devices were incubated for $15 \mathrm{~min}$ at $37^{\circ} \mathrm{C}$ in the diluted dye solution, briefly rinsed to remove excess dye, and incubated at $37^{\circ} \mathrm{C}$ in fresh media for an additional $30 \mathrm{~min}$ before imaging.

\section{Fluorescence Microscopy}

Brightfield and fluorescence images were captured on a Zeiss 200 Axiovert microscope with an AxioCam MRm digital camera, typically using a 2.5x objective and 1.6x optovar for full images of a single array. JC-1 fluorescence was measured using Zeiss \#38 and \#31 filter sets for the green and red channels. The green fluorescence channel represents the overall loading of JC-1, while the red channel represents the shift in fluorescence that occurs with the potential dependent formation of J-aggregates at high JC-1 concentrations in the mitochondrial membranes [19,23]. Rh123 fluorescence was measured using a Zeiss \#38 filter set. Exposure times were selected to maximize the dynamic range of the resulting images. A brightfield image was taken along with each set of fluorescence images for alignment, quality control, and display purposes.

\section{Cryopreservation and Recovery}

After imaging, cells were allowed to recover for $30 \mathrm{~min}$ in a $37^{\circ} \mathrm{C}$ incubator. Freezing solutions were prepared using HypoThermosol-Base (BioLife Solutions) with $10 \% \mathrm{Me}_{2} \mathrm{SO}$ added [24]. The devices were quickly transferred into $1.5 \mathrm{ml}$ Nalgene cryovials filled with the freezing solution, placed on ice for a short time for cell equilibration with the cryoprotectant, and placed in a controlled-rate freezer (Planer KRYO 10). The devices were first cooled to $-6^{\circ} \mathrm{C}$, at which time extracellular ice was seeded by the applying cold forceps to the outside of each vial. This was followed by a 10 minute holding period to allow for ice growth and temperature equilibration. The devices were then cooled at a rate of $-5^{\circ} \mathrm{C} / \mathrm{min}$ to $-80^{\circ} \mathrm{C}$, plunged into liquid nitrogen, and stored for up to a week. After the storage period, the vials were thawed rapidly by gentle agitation for $2 \mathrm{~min}$ in a $37^{\circ} \mathrm{C}$ water bath. The devices were then rinsed in warm $\mathrm{C}$ $+\mathrm{H}$, transfered to fresh medium, and allowed to recover at $37^{\circ} \mathrm{C}$ for $1 \mathrm{hr}$. 


\section{Viability Assays}

Stock solutions of $1 \mathrm{mM}$ Sytox Blue (Molecular Probes) were diluted 1:1000 in $\mathrm{C}+\mathrm{H}$ to a final concentration of $1 \mu \mathrm{M}$ and used in combination with JC-1 stained cells. Stock solutions of 2 $\mathrm{mM}$ ethidium homodimer-1 (Molecular Probes) were diluted 1:1000 in $\mathrm{C}+\mathrm{H}$ to a final concentration of $2 \mu \mathrm{M}$, and used in combination with Rh123 stained cells. After recovering from cryopreservation, devices were transferred into $35 \mathrm{~mm}$ dishes containing one of the diluted dyes and incubated at $37^{\circ} \mathrm{C}$ for $5 \mathrm{~min}$ before imaging. Sytox Blue fluorescence was measured using a Zeiss \#5 filter set, while EthD-1 was measured using a Zeiss \#31 filter set. JC-1 or Rh123 fluorescence images were usually taken at the same time.

\section{Image Processing and Statistical Analysis}

A set of custom software tools were developed for automated image processing, quality control, and preparing convenient data sets for later analysis. This software is described in detail elsewhere and available upon request [21]. Plotting and statistical analysis were performed using the R programming environment [18].

\section{Results}

Primary hepatocytes were seeded in the microwell arrays, stained with a $\Delta \Psi$-sensitive dye, imaged, cryopreserved, and assessed for viability. The result of each processing step is shown for a typical array in Figure 1. Data analysis centered on correlating the initial mitochondrial membrane potential, as represented by JC- 1 or Rh123 fluorescence, with short term survival after cryopreservation.

\section{JC-1 and Rh123 Fluorescence Distributions}

The hepatocytes were stained with either JC- 1 or Rh123 and imaged before cryopreservation. Using the microwell array cytometry system, it is easy to create data products similar to those generated by flow cytometry. A scatter plot of the observed red and green JC-1 fluorescence intensities is included in Panel A of Figure 2. Two distinct subpopulations can be seen in the panel, one with high JC-1 green fluorescence and one with much lower fluorescence. A histogram of the green channel values alone (not shown) was found to have a bimodal distribution, with a clear separation between the two hepatocyte subpopulations. Cell counts and array overlay images showed that cells from the two subpopulations were uniformly distributed both between the arrays and spatially across each array.

The significance of these subpopulations is not clear from this experiment alone, but may represent cells from different regions of the liver. Despite the bimodal peak of the JC-1 green channel, and a clear difference between the mean JC-1 red intensity in the two subpopulations, the JC-1 red and Rh123 intensity distributions did not resolve into multiple peaks. Since these channels are primarily sensitive to $\Delta \Psi$, high variability in this factor may overwhelm any systematic differences between the subpopulations [4].

\section{Live-Dead Assays}

Live-dead assays were performed after cryopreservation using the retained Rh123 or JC- 1 to indicate live cells and a membrane impermeant nucleic acid stain added after thawing to indicate dead cells. For fluorescence channel compatibility, ethidium homodimer was used with the Rh123 stained cells and Sytox Blue was used with the JC-1 stained cells. The results of these viability assays are plotted in the remaining panels of Figure 2. In both cases there is good separation between the live and dead cell populations. The rectangular regions in Panels $\mathrm{C}$ and $\mathrm{D}$ represent the gates that were used to designate live cells, dead cells, and cells lost during thawing. The trimodal distribution seen with the nucleic acid stains in Panel B reflect 
the fact that primary hepatocytes can be either mono or binucleated. Overall viabilities after cryopreservation for the JC- 1 and Rh123 experiments were 56.4\% and $47.2 \%$ respectively, with $4.8 \%$ and $4.6 \%$ of the cells lost from the array. These results are somewhat lower than those of similar bulk experiments with suspended cells [24].

\section{Effect of Mitochondrial Membrane Potential on Cryopreservation Outcomes}

The effect of initial $\Delta \Psi$ on cryopreservation outcomes was investigated by combining the prepreservation Rh123 or JC-1 intensity data with the post-preservation viability data. Using the viability data from each experiment, the hepatocytes were classified into three different groups: cells that survived preservation, cells that died during preservation, and cells lost from the device. Histograms of the pre-preservation Rh123 and JC-1 intensity data were then plotted for each of these groups and compared.

The resulting histograms are shown in the top row of Figure 3 for JC- 1 red, JC- 1 green, and Rh123. Subtle differences can be observed in the intensity distributions for each population. Most strikingly, the JC-1 red and Rh123 intensity distributions are shifted to the left for cells that go on to survive preservation. Though the difference appears small, the number of cell samples is large and unpaired t-tests showed that the mean JC-1 red and Rh123 intensities are significantly smaller in cells that went on to survive preservation compared to those that did not survive preservation ( $\mathrm{p} \ll 0.01$ in both cases). This trend was not observed in the JC-1 green channel and the t-test did not show a significant difference in the mean intensity between the groups $(\mathrm{p}=0.195)$.

The effect of the intensity shift can be better appreciated by plotting percent viability as a function of initial fluorescence intensity as shown in the bottom row of Figure 3. In the case of JC- 1 red and Rh123, the viability is approximately $60 \%$ at low fluorescence intensities and drops to approximately $40 \%$ at high intensities. Logistic regression of cell survival on initial fluorescence intensity showed highly significant negative correlations for JC-1 red and Rh123 ( $\mathrm{p} \ll 0.01)$, but was not significant for JC-1 green $(\mathrm{p}=0.189)$. The significant negative correlation with viability suggests that having a high mitochondrial membrane potential before cryopreservation is detrimental to the cells and leads to worse outcomes. However, the relatively small magnitude of the effect suggests that other factors also play important roles.

\section{Multidimensional Analysis}

Since both red and green channel intensities were acquired for each cell in the JC-1 experiment, it is possible to examine cell viability across the 2D landscape of initial JC-1 fluorescence values rather than look at each channel separately as done above. Figure 4 shows the variation in cell survival rates seen across the landscape of JC-1 intensities, with green representing an upper limit of $70 \%$ viability and red representing a lower limit of $20 \%$ viability. Live cell and total cell density estimates were computed using a Gaussian kernel and the ratio of these density estimates was plotted. Several features are apparent in the joint distribution that are not obvious in the single channel plots, such as the poor viability for cells with low JC-1 red fluorescence but high JC-1 green fluorescence. Multidimensional analyses of this type are made possible in part by the high cell counts achievable with microwell array cytometry, without which there would be insufficient data for reliable viability estimates in regions with low cell density.

\section{Discussion}

The dual goals of this project were to investigate the role of mitochondrial state in determining cryopreservation outcomes and to introduce microwell array cytometry as a high-throughput, high-content platform for single cell studies in the field of biopreservation. Given the positive 
results of the initial experiments, we expect the system will be used in the future to investigate a wide range of cellular features that may impact preservation outcomes.

The experiments described in this paper focused on the role of mitochondrial membrane potential in the cryopreservation of primary hepatocytes. Based on current literature, it was unclear how the mitochondrial state of a cell would affect the outcome of preservation. On the one hand, cells with a high mitochondrial membrane potential $(\Delta \Psi)$ have been shown to be more resistant to a number of death-inducing stimuli [6]. On the other hand, studies of freeze and desiccation tolerant organisms have shown an almost universal tendency to drastically reduce metabolic activity and oxygen consumption prior to the onset of the stress [8,25]. Tolerant organisms are also known to have numerous mitochondrial adaptations that inhibit calcium leakage and apoptosis [9-11].

Our results with JC-1 and Rh123 staining suggest that a moderately suppressed mitochondrial membrane potential may be beneficial in cryopreservation, but is clearly not the sole determinant of outcome. In interpreting this result, it should be noted that the membrane potential is not a direct measure of mitochondrial activity. In healthy mitochondria, a low mitochondrial membrane potential can be the result of increased oxidative phosphorylation, whereas a high membrane potential can occur under resting (state 4) conditions, where most respiration is devoted to the compensation of proton leak rather than ATP synthesis [16]. In contrast, a total collapse of $\Delta \Psi$, which was not observed here, is often seen upon the opening of the mitochondrial permeability transition pore during apoptotic signaling [1]. Activation of the transition pore involves compromise of inner membrane integrity and thus of membrane potential. This phenomenon is distinct from mitochondrial outer membrane permeabilization via Bax/Bak poration, which does not necessarily alter the membrane potential [2]. Given these complexities, caution should be exercised when interpreting $\Delta \Psi$ data alone in the absence of other mechanistic information. Further exploration of mitochondrial state using the microwell image cytometry system will help address these questions.

As mentioned in the results, the overall viability with the devices was somewhat lower but comparable to what we typically achieve with suspended hepatocytes. Under the right circumstances, the use of microwell arrays could actually be beneficial, since the large physical forces involved in freezing or drying can easily displace cells attached to a flat surface or crush suspended cells between growing ice crystals [7]. In many of the freezing experiments, it was observed that any cells attached to the array, but not protected by a well were either killed or ripped away from the surface during preservation. The freezing protocol used for the arrays was directly adapted from our protocol for suspended primary hepatocytes and not independently optimized. Viability on the devices was nevertheless quite good and could potentially exceed our best results for suspended cells with additional work. One significant problem that needs to be addressed is bubble formation on the device during thawing, which sometimes killed significant numbers of cells and interfered with image analysis. We are currently working on several strategies to eliminate this problem, such as adding a cellcompatible surfactant like Poloxamer-188 and adjusting the thawing protocol [12].

Another potential cause of the reduced overall viability is cytotoxicity or freeze-sensitization caused by the mitochondrial dyes. Since the dyes accumulate in the mitochondria at very high concentrations, this is a reasonable concern. Based on our unpublished observations, however, Rh123 and JC-1 appear to have minimal cytotoxicity to primary rat hepatocytes and little effect on cryopreservation outcomes. Seeded arrays stained with either dye but not frozen had nearly $100 \%$ viability after several days in culture, similar to arrays not exposed to the dye.

Additionally, when frozen in suspension, stained hepatocytes did not have significantly lower viability than unstained hepatocytes, even when high concentrations of the dyes were included in the freezing medium. 
A number of other improvements could potentially be made to the microwell system and future experimental designs. One weakness of the current experiments was the use of short term viability as the only cryopreservation endpoint. In future experiments, this will be addressed by looking at cell viability again after one or more days and using apoptosis assays to determine the type of cell death that has occurred. Additional fluorescent dyes and GFP reporter constructs will also be used to study factors such as free radical production, calcium release, caspase activity, and stress response pathway activation. A number of GFP reporter constructs sensitive to key transcription factors have previously been developed in our lab and will be used with the microwell system [30].

Long term viability and function is an important issue in cryopreservation, particularly with primary cells. Previous work in our laboratory and others has explored how cryopreservation protocols and culture conditions affect the long term behavior of hepatocytes [5,24,27,28]. Ideally, hepatocyte viability and function would be monitored for up to several weeks in the arrays. Unfortunately, long term endpoints such as these are not currently feasible for several reasons. Primary hepatocytes are very sensitive to culture conditions and long term seeding in microwells without cell-to-cell contact is far from ideal. In seeding experiments without freezing, viability remains near $100 \%$ for several days, but later drops to almost zero. With cell lines, long term endpoints are complicated by cell division and movement, which disrupt the physical separation and spatial organization of the cells in the device. We are currently working on several potential ways to address these problems and extend the length of time cells can be kept in the arrays, such as improving the surface coatings of the device or embedding the microwells in a thin Matrigel layer after cell seeding, which has been shown to maintain hepatocyte function in the absence of cell-cell contact [13].

With conventional methods such as flow cytometry or manual image analysis, it is time consuming and difficult to reliably track large numbers of individual cells through the preservation and recovery process. As a result, relatively little is known about how heterogeneity in the bioenergetic, metabolic, transcriptional, or signaling phenotypes of a cell population affects preservation outcomes. With microwell array cytometry, these difficulties are largely eliminated, transforming cell population heterogeneity from unwanted noise into a source of useful experimental variation. This variation can then be harnessed to study the phenotypic determinants that affect preservation outcomes and used to identify protective and maladaptive cell responses to target for intervention. Based on the results of this study, we believe that microwell array cytometry will be an extremely powerful and valuable tool for the study of biopreservation damage mechanisms and the development of new approaches in biopreservation.

\section{Acknowledgments}

Funding from NIH (P41 EB002503, R01 GM071345, R01 AI063795, R01 DK046270, R01 DK043371) and Shriners Hospitals for Children (\#8450, 8460, 8490, 8650) are gratefully acknowledged.

\section{References}

1. Bernardi P, Krauskopf A, Basso E, Petronilli V, Blachly-Dyson E, Di Lisa F, Forte MA. The mitochondrial permeability transition from in vitro artifact to disease target. FEBS J 2006;273(10): 2077-2099. [PubMed: 16649987]

2. Chipuk JE, Bouchier-Hayes L, Green DR. Mitochondrial outer membrane permeabilization during apoptosis: the innocent bystander scenario. Cell Death and Differentiation 2006;13(8):1396-1402. [PubMed: 16710362]

3. Crowe JH, Crowe LM, Oliver AE, Tsvetkova N, Wolkers W, Tablin F. The trehalose myth revisited: introduction to a symposium on stabilization of cells in the dry state. Cryobiology 2001;43(2):89-105. [PubMed: 11846464] 
4. Duchen MR, Surin A, Jacobson J. Imaging mitochondrial function in intact cells. Methods Enzymol 2003;3(61):353-389. [PubMed: 12624920]

5. Dunn JC, Tompkins RG, Yarmush ML. Long-term in vitro function of adult hepatocytes in a collagen sandwich configuration. Biotechnol. Prog 1991;7(3):237-245. [PubMed: 1367596]

6. Follstad BD, Wang DI, Stephanopoulos G. Mitochondrial membrane potential differentiates cells resistant to apoptosis in hybridoma cultures. Eur. J. Biochem 2000;267(22):6534-6540. [PubMed: 11054104]

7. Fowler A, Toner M. Cryo-injury and biopreservation. Ann. N Y Acad. Sci 2005;1066:119-135. [PubMed: 16533923]

8. Hand SC. Quiescence in Artemia franciscana embryos: reversible arrest of metabolism and gene expression at low oxygen levels. J. Exp. Biol 1998;201(Pt 8):1233-1242. [PubMed: 9510534]

9. Hand SC, Menze MA. Mitochondria in energy-limited states: mechanisms that blunt the signaling of cell death. J. Exp. Biol 2008;211(Pt 12):1829-1840. [PubMed: 18515712]

10. Menze MA, Hutchinson K, Laborde SM, Hand SC. Mitochondrial permeability transition in the crustacean Artemia franciscana: absence of a calcium-regulated pore in the face of profound calcium storage. Am. J. Physiol. Regul. Integr. Comp. Physiol 2005;289(1):R68-R76. [PubMed: 15718386]

11. Menze MA, Hand SC. Caspase activity during cell stasis: avoidance of apoptosis in an invertebrate extremophile, Artemia franciscana. Am. J. Physiol. Regul. Integr. Comp. Physiol 2007;292 (5):R2039-R2047. [PubMed: 17255212]

12. Merchant FA, Holmes WH, Capelli-Schellpfeffer M, Lee RC, Toner M. Poloxamer 188 enhances functional recovery of lethally heat-shocked fibroblasts. J. Surg. Res 1998;74(2):131-140. [PubMed: 9587351]

13. Moghe PV, Coger RN, Toner M, Yarmush ML. Cell-cell interactions are essential for maintenance of hepatocyte function in collagen gel but not on matrigel. Biotechnol Bioeng 1997;56(6):706-711. [PubMed: 18642343]

14. Nahmias Y, Berthiaume F, Yarmush ML. Integration of technologies for hepatic tissue engineering. Adv. Biochem. Eng. Biotechnol 2007;103:309-329. [PubMed: 17195468]

15. Newmeyer DD, Ferguson-Miller S. Mitochondria: releasing power for life and unleashing the machineries of death. Cell 2003;112(4):481-490. [PubMed: 12600312]

16. Nicholls, DG.; Ferguson, SJ. Bioenergetics 3. New York: Academic Press; 2002. p. 297

17. Pepperkok R, Ellenberg J. High-throughput fluorescence microscopy for systems biology. Nat. Rev. Mol. Cell. Biol 2006;7(9):690-696. [PubMed: 16850035]

18. R Development Core Team. R: A Language and Environment for Statistical Computing. Vienna: R Foundation for Statistical Computing; 2007.

19. Reers M, Smiley ST, Mottola-Hartshorn C, Chen A, Lin M, Chen LB. Mitochondrial membrane potential monitored by JC-1 dye. Methods Enzymol 1995;260:406-417. [PubMed: 8592463]

20. Reynolds JA, Hand SC. Differences in isolated mitochondria are insufficient to account for respiratory depression during diapause in artemia franciscana embryos. Physiol Biochem Zool 2004;77(3):366377. [PubMed: 15286911]

21. Roach KL, King K, Kohane IS, Yarmush ML, Toner M. High Throughput Single Cell Bioinformatics. (submitted)

22. Shapiro HM. Membrane potential estimation by flow cytometry. Methods 2000;21(3):271-279. [PubMed: 10873481]

23. Smiley ST, Reers M, Mottola-Hartshorn C, Lin M, Chen A, Smith TW, Steele GD Jr, Chen LB. Intracellular heterogeneity in mitochondrial membrane potentials revealed by a J-aggregate-forming lipophilic cation JC-1. Proc. Natl. Acad. Sci. U. S. A 1991;88(9):3671-3675. [PubMed: 2023917]

24. Sosef MN, Baust JM, Sugimachi K, Fowler A, Tompkins RG, Toner M. Cryopreservation of isolated primary rat hepatocytes: enhanced survival and long-term hepatospecific function. Ann. Surg 2005;241(1):125-133. [PubMed: 15622000]

25. Storey KB, Storey JM. Metabolic rate depression in animals: transcriptional and translational controls. Biol. Rev. Camb. Philos. Soc 2004;79(1):207-233. [PubMed: 15005178]

26. Storey KB. Reptile freeze tolerance: metabolism and gene expression. Cryobiology 2006;52(1):116. [PubMed: 16321368] 
27. Sugimachi K, Sosef MN, Baust JM, Fowler A, Tompkins RG, Toner M. Long-term function of cryopreserved rat hepatocytes in a coculture system. Cell Transplant 2004;13(2):187-195. [PubMed: 15129765]

28. Sugimachi K, Roach KL, Rhoads DB, Tompkins RG, Toner M. Nonmetabolizable glucose compounds impart cryotolerance to primary rat hepatocytes. Tissue Eng 2006;12(3):579-588. [PubMed: 16579691]

29. Tunnacliffe A, Lapinski J. Resurrecting Van Leeuwenhoek's rotifers: a reappraisal of the role of disaccharides in anhydrobiosis. Philos. Trans. R. Soc. Lond. B. Biol. Sci 1438;358:1755-1771. [PubMed: 14561331]

30. Wieder KJ, King KR, Thompson DM, Zia C, Yarmush ML, Jayaraman A. Optimization of reporter cells for expression profiling in a microfluidic device. Biomed Microdevices 2005;7(3):213-222. [PubMed: 16133809] 

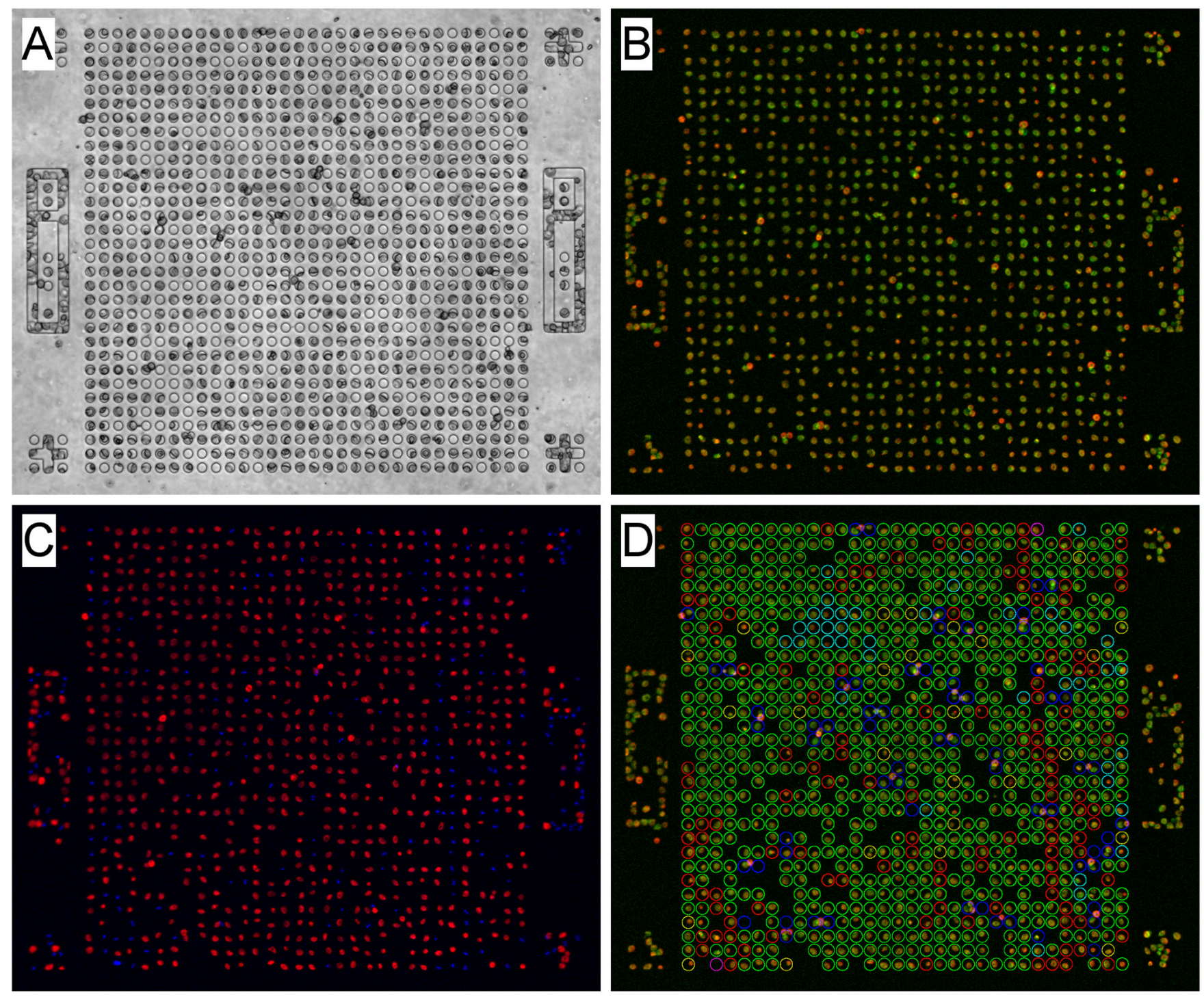

Figure 1.

The microwell array design. Panel A is a brightfield image taken after seeding hepatocytes in the 32-by-32 grid of microwells. Alignment features and identification features are included on each side. In Panel B the cells have been stained with the mitochondrial dye JC-1 before cryopreservation and imaged in the red and green fluorescence channels. Panel $\mathrm{C}$ shows the results of Live/Dead staining after cryopreservation, with red representing polarized mitochondria in live cells and blue representing nuclear staining in dead cells. Panel D was generated using the viability data and displays the eventual fate of each cell on top of the original JC-1 fluorescence image for rapid visual inspection. Green $=$ survival, Red $=$ death, Yellow $=$ lost from well, Magenta $=$ uncertain, Blue or Cyan $=$ debris around well . 

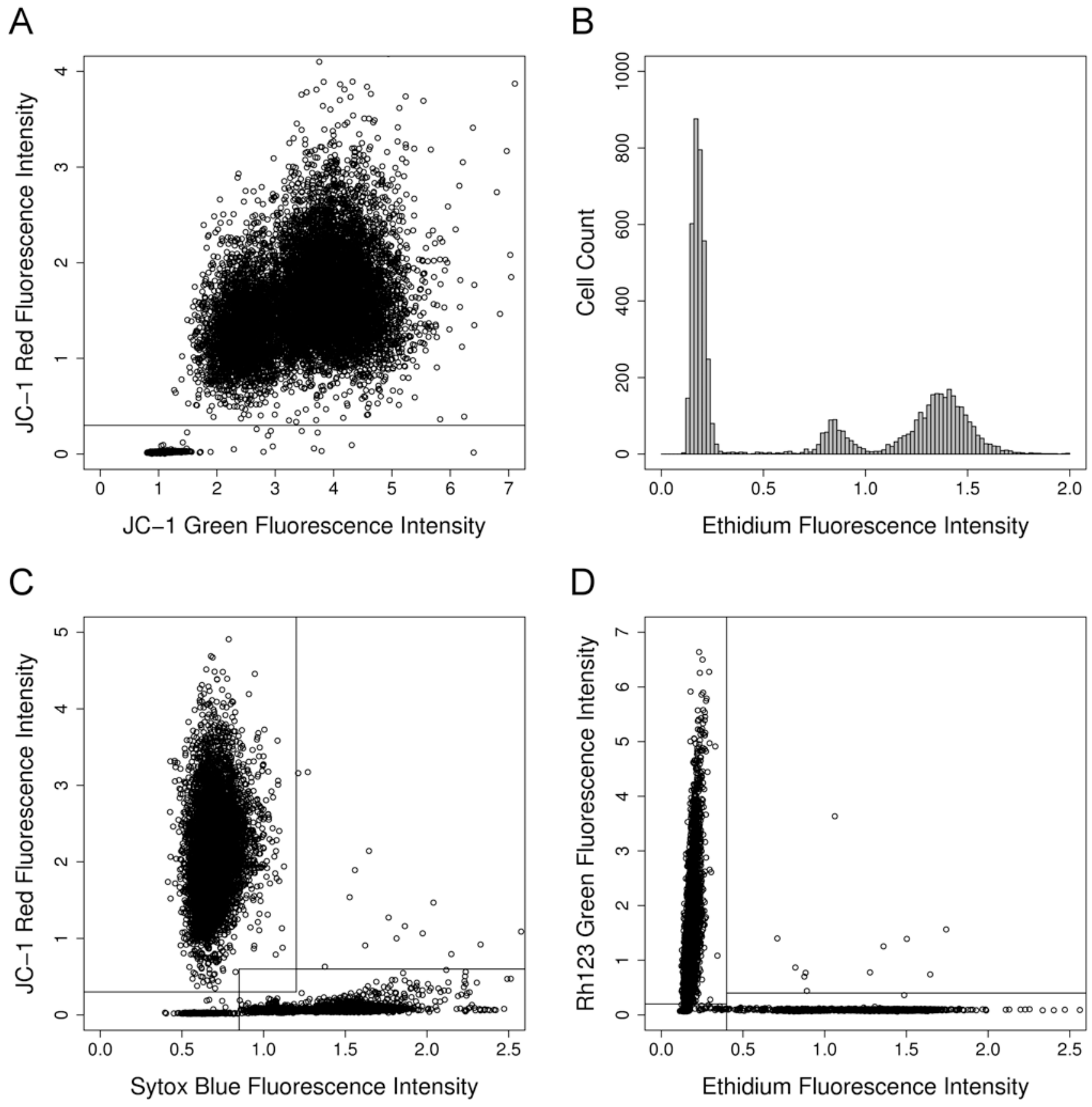

Figure 2.

Data analysis after image processing. Panel A shows the distribution of JC-1 red and green channel fluorescence intensities for approximately 10,000 cells in a 16-array device. Panel B shows the intensity histogram of a membrane impermeant nuclear stain used in the viability assays. The two peaks correspond to mono and binucleated hepatocytes. Panels C and D illustrate the gating used to identify live and dead cells after cryopreservation. 
A

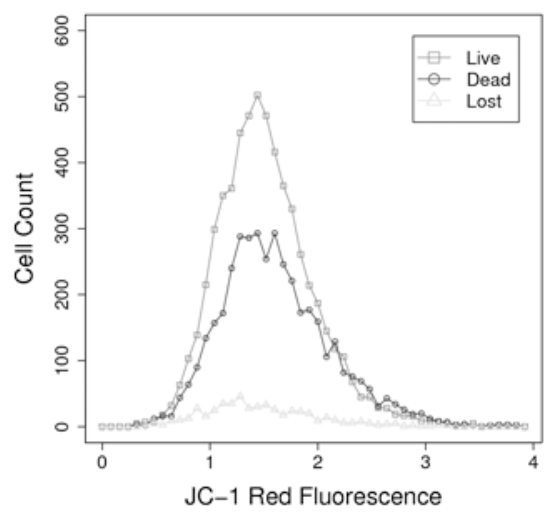

D

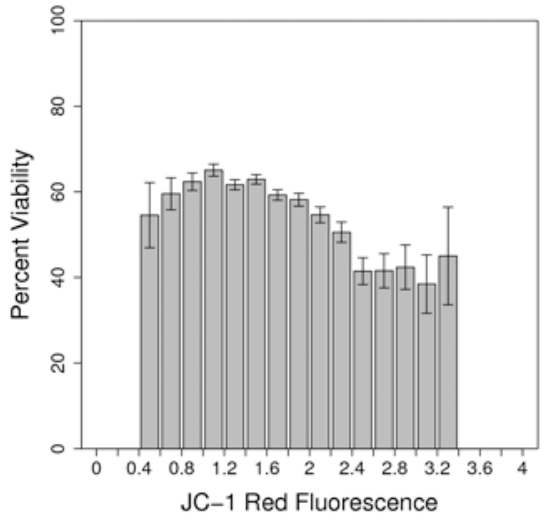

B

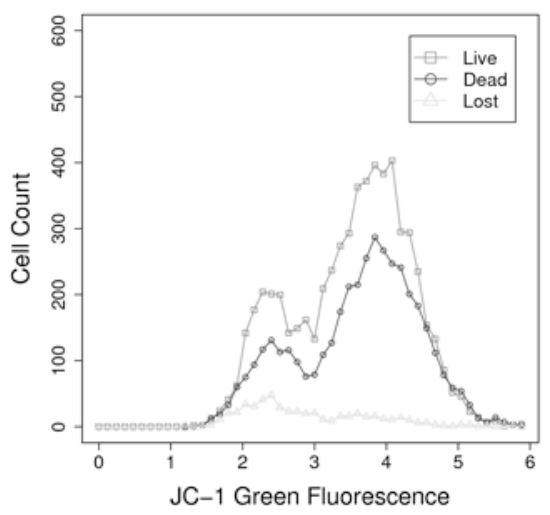

E

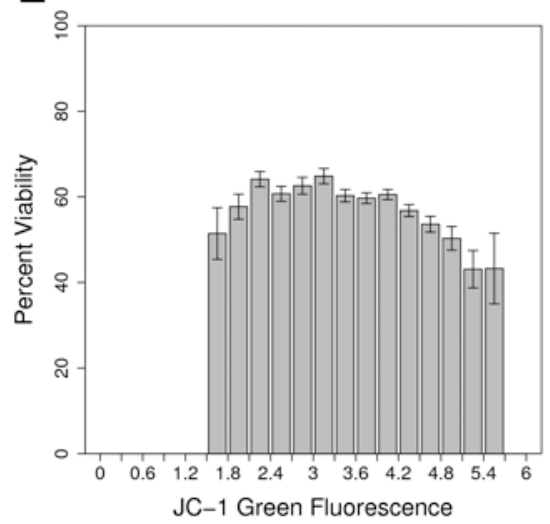

C

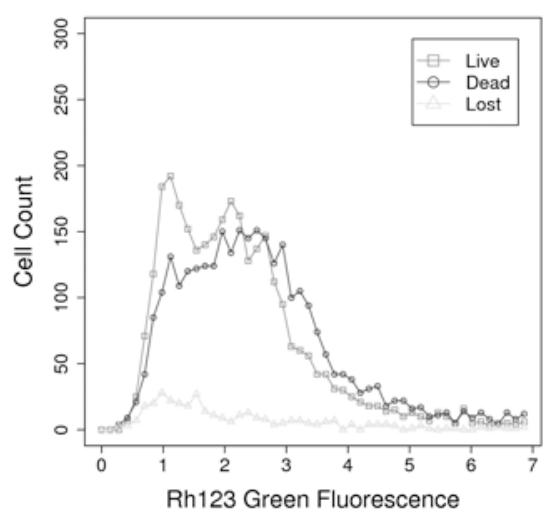

F

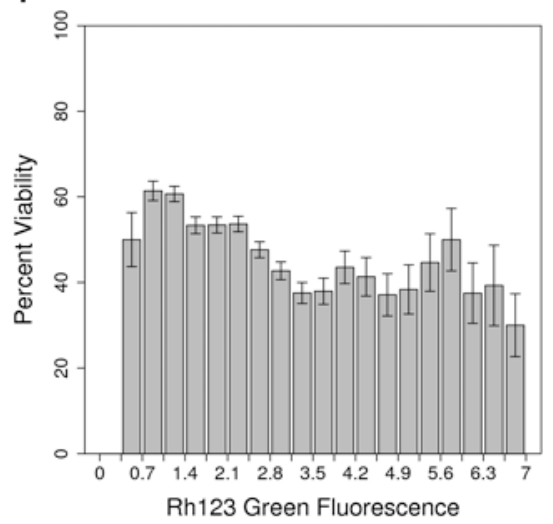

Figure 3.

Hepatocyte viability after cryopreservation was plotted as a function of JC-1 and Rh123 fluorescence intensity as a proxy for mitochondrial membrane potential. Hepatocytes were separated into three classes: cells that survived, cells that died, and cells that were lost from the array. Top row: cell counts in each class for each of three fluorescence channels. Bottom row: percent viability plotted as a function of fluorescence intensity. The error bars represent the standard error of the percent viability given the number of cells in that bin. 


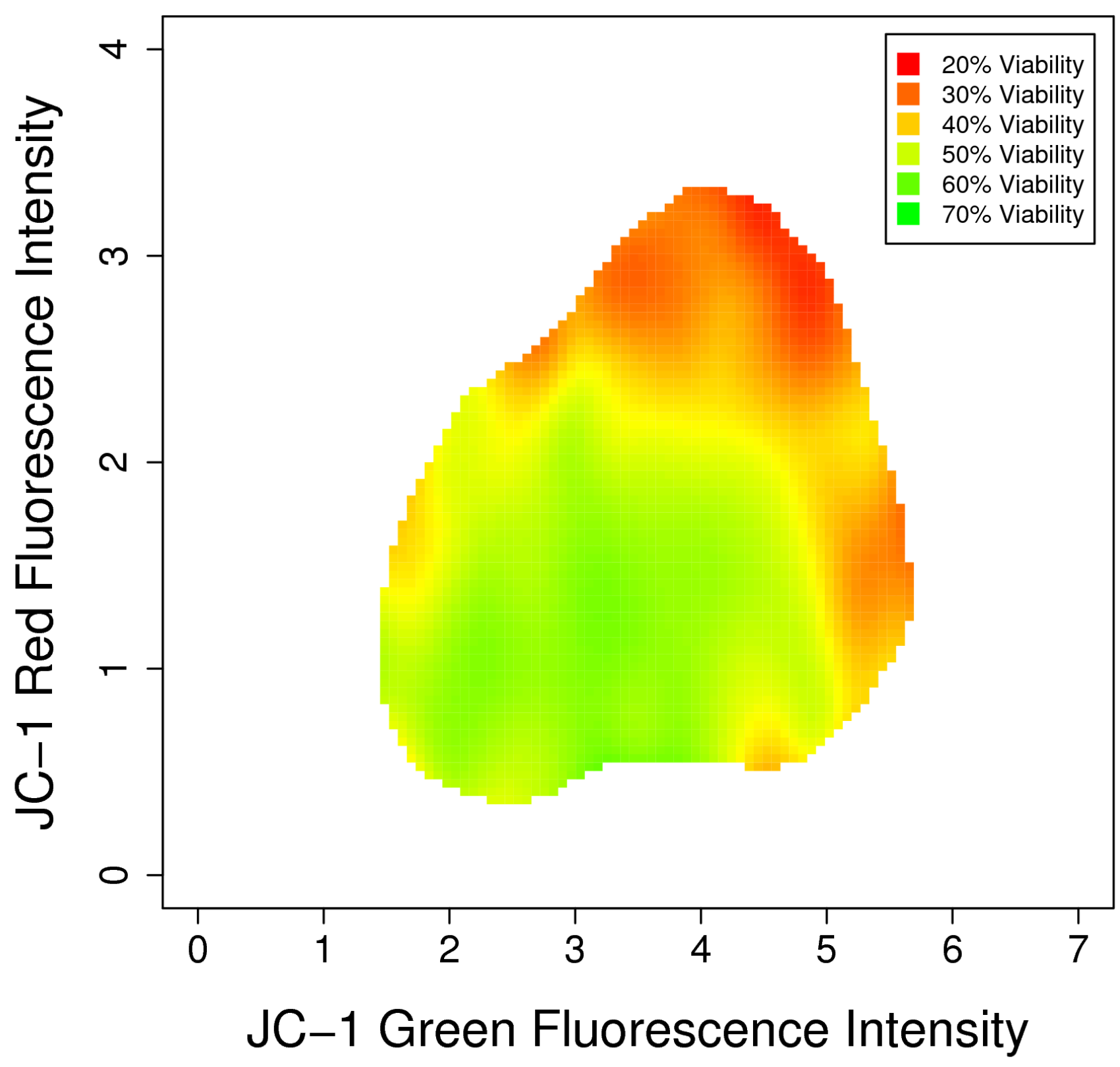

Figure 4.

Survival rates vary across the landscape of initial JC-1 fluorescence values, with green representing a high of $70 \%$ viability and red representing a low of $20 \%$ viability. The high cell counts and measurement capabilities of microwell array cytometry allow preservation outcomes to be mapped across a multidimensional landscape of cell phenotypes with a single experiment. 